\title{
On a Bernoulli shift with non-identical factor measures
}

\author{
TOSHIHIRO HAMACHI \\ Department of Mathematics, Kyushu University, \\ Ropponmatsu, Chuo-Ku, Fukuoka 810, Japan
}

(Received 4 June 1981)

Abstract. There exists a Bernoulli shift with non-identical factor measures for which no invariant $\sigma$-finite equivalent measure exists.

\section{Introduction}

Our purpose is to give an example of a Bernoulli shift $T$ acting on an infinite product measure space

$$
(\Omega, \mathscr{F}, P)=\left(\prod_{-\infty<k<\infty}\{0,1\}, \underset{-\infty<k<\infty}{\bigvee} \mathscr{F}_{k}, \prod_{-\infty<k<\infty} P_{k}\right)
$$

such that the shift $T$,

$$
(T \omega)_{k}=\omega_{k+1} \text { for } \omega=\left(\omega_{k}\right)_{-\infty<k<\infty},
$$

is non-singular, i.e. $P(A)=0$ if and only if $P(T A)=0$ for $A \in \mathscr{F}=V_{-\infty<k<\infty} \mathscr{F}_{k}$ such that $T$ is ergodic and there exists no shift-invariant $\sigma$-finite measure equivalent to the infinite product measure $P=\prod_{-\infty<k<\infty} P_{k}$, where $P_{k}$ is a probability measure on the set $\{0,1\}$ and $\mathscr{F}_{k}$ is the smallest $\sigma$-algebra which makes the $k$ th coordinate $\omega_{k}$ of $\omega \in \Omega$ measurable.

This problem was raised by U. Krengel at the symposium on Ergodic Theory at Oberwolfach 1978. He gave in [3] an ergodic Bernoulli shift without finite invariant measure.

In $\S 2$ we give non-ergodic Bernoulli shifts which are dissipative. In $\S 3$ we give an ergodic Bernoulli shift without $\sigma$-finite invariant measure.

\section{Dissipative Bernoulli shifts}

Let $\Omega=\prod_{-\infty<k<\infty}\{0,1\}$, and $T$ be the shift on $\Omega$, i.e.

$$
(T \omega)_{k}=\omega_{k+1} .
$$

Take a probability measure

$$
P=\prod_{-\infty<k<\infty} P_{k}
$$

with

$$
\begin{aligned}
P_{k}(0)=P_{k}(1) & =\frac{1}{2} \quad(k \geq 0) \\
0<P_{k}(0)<1, \quad P_{k}(1) & =1-P_{k}(0) \quad(k<0) .
\end{aligned}
$$


It follows from Kakutani's theorem on the equivalence of infinite product measures [2] that $T$ is non-singular if and only if

$$
\sum_{-\infty<k<\infty} P_{k-1}(0) P_{k}(0)\left(\left(P_{k-1}(1) / P_{k-1}(0)\right)^{\frac{1}{2}}-\left(P_{k}(1) / P_{k}(0)\right)^{\frac{1}{2}}\right)^{2}<\infty
$$

In this case we have that the Radon-Nikodym derivative $d P T / d P$ of the measure $P T$,

$$
(P T)(A)=P(T A) \text { for } A \in \mathscr{F},
$$

with respect to the measure $P$ is given by

$$
(d P T / d P)(\omega)=\prod_{-\infty<k<\infty} P_{k-1}\left(\omega_{k}\right) / P_{k}\left(\omega_{k}\right)
$$

for a.e. $\omega$, where the infinite product converges almost everywhere. In [3] Krengel claimed that the shift $T$ is dissipative if

$$
P_{k}(1) / P_{k}(0)=3 \quad(k<0)
$$

In fact, more generally we have:

THEOREM 1. Let $P_{k}(0)=P_{k}(1)=\frac{1}{2}(k \geq 0)$ and let $P_{k}(1) / P_{k}(0)=\lambda$ ( $\lambda$ is a constant $>0)(k<0)$. If $\lambda \neq 1$ then the shift $T$ on $\Omega=\prod_{-\infty<k<\infty}\{0,1\}$ is dissipative.

Proof. The non-singularity condition (1) for the shift $T$,

$$
\begin{aligned}
\sum_{-\infty<k<\infty} P_{k-1}(0) P_{k}(0)\left(\left(P_{k-1}(1) / P_{k-1}(0)\right)^{\frac{1}{2}}-\left(P_{k}(1) / P_{k}(0)\right)^{\frac{1}{2}}\right)^{2} \\
\quad=P_{-1}(0) P_{0}(0)\left(\left(P_{-1}(1) / P_{-1}(0)\right)^{\frac{1}{2}}-\left(P_{0}(1) / P_{0}(0)\right)^{\frac{1}{2}}\right)^{2} \\
\quad=\left(\lambda^{\frac{1}{2}}-1\right)^{2} /(2(1+\lambda)) \\
\quad<\infty
\end{aligned}
$$

is satisfied, and we have from (2)

$$
\left(d P T^{n} / d P\right)(\omega)=(2 /(1+\lambda))^{n} \lambda^{s_{n}(\omega)} \quad(n \geq 1),
$$

where $S_{n}(\omega)=\omega_{0}+\omega_{1}+\cdots+\omega_{n-1}$.

What we are going to prove is that the infinite series

$$
\sum_{n=1}^{\infty}(2 /(1+\lambda))^{n} \lambda^{s_{n}(\omega)}
$$

converges a.e. $\omega$. Take $0<\theta<\frac{1}{2}$, then a standard fact says that

$$
\lim _{n \rightarrow \infty}\left(S_{n}(\omega)-\frac{1}{2} n\right) /(n / 4)^{\frac{1}{2}+\theta}=0 \quad \text { a.e. } \omega .
$$

We assume $\lambda>1$. For any $\varepsilon>0$ and for a.e. $\omega$, all but a finite number of $n$ satisfy

$$
-\varepsilon<\left(S_{n}(\omega)-\frac{1}{2} n\right) /(n / 4)^{\frac{1}{2}+\theta}<\varepsilon .
$$

Then we have for all large $n$

$$
\begin{aligned}
(2 /(1+\lambda))^{n} \lambda^{S_{n}(\omega)}<\exp & \left\{n \log (2 /(1+\lambda))+n \log (\lambda) / 2+\varepsilon \log (\lambda)(n / 4)^{\frac{1}{2}+\theta}\right\} \\
= & \exp \left\{n^{\frac{1}{2}}\left(\log \left(2 \lambda^{\frac{1}{2}} /(1+\lambda)\right) n^{\frac{1}{2}}+\varepsilon \log (\lambda)(n / 4)^{\theta}\right)\right\} .
\end{aligned}
$$

Since for all large $n$

$$
\log \left(2 \lambda^{\frac{1}{2}} /(1+\lambda)\right) n^{\frac{1}{2}}+\varepsilon \log (\lambda)(n / 4)^{\theta}<-1,
$$


we have for all large $n$

$$
(2 /(1+\lambda))^{n} \lambda^{S_{n}(\omega)}<\exp \left(-n^{\frac{1}{2}}\right)
$$

Since the series

$$
\sum_{n=1}^{\infty} \exp \left(-n^{\frac{1}{2}}\right)
$$

converges, the theorem is proved if $\lambda>1$. If $\lambda<1$, it is enough to see that for a.e. $\omega$ and for all large $n$

$$
(2 /(1+\lambda))^{n} \lambda^{S_{n}(\omega)}<\exp \left\{n^{\frac{1}{2}}\left[\left(\log \left(2 \lambda^{\frac{1}{2}} /(1+\lambda)\right) n^{\frac{1}{2}}-\varepsilon \log (\lambda)(n / 4)^{\theta}\right]\right\} .\right.
$$

\section{Bernoulli shift without $\sigma$-finite invariant measure}

We are concerned with a class of infinite product measures

on

$$
P=\prod_{-\infty<k<\infty} P_{k}
$$

$$
\Omega=\prod_{-\infty<k<\infty}\{0,1\}
$$

given by

where

$$
P_{k}= \begin{cases}\mu & \text { if } k \geq 0 \\ \nu_{t} & \text { if }-N_{t}<k \leq-M_{t-1} \\ \mu & \text { if }-M_{t}<k \leq-N_{t} \quad(t \geq 1)\end{cases}
$$

$$
N_{t}=M_{t-1}+n_{t}, \quad M_{t}=N_{t}+m_{t}, \quad M_{0}=1,
$$

$n_{t}$ and $m_{t}$ are positive integers, and

$$
\begin{gathered}
\mu(0)=\mu(1)=\frac{1}{2}, \quad \nu_{t}(0)=1 /\left(1+\lambda_{t}\right), \quad \nu_{t}(1)=\lambda_{t} /\left(1+\lambda_{t}\right), \\
\lambda_{1}>\lambda_{2}>\cdots>\lambda_{t}>1 \quad(t \geq 1) .
\end{gathered}
$$

We shall also consider the measure

$$
Q=\prod_{-\infty<k<\infty} Q_{k}
$$

with $Q_{k}=\mu(k \in \mathbb{Z})$. The non-singular condition (1) for the shift $T$ on $(\Omega, P)$ defined above is equivalent to the condition

$$
\sum_{i=1}^{\infty}\left(\log \left(\lambda_{t}\right)\right)^{2}<\infty
$$

What we are going to do is to give inductively a sequence $\left(\lambda_{t}, n_{t}, m_{t}\right)_{t \geq 1}$ such that the shift $T$ is non-singular, ergodic and admits no invariant $\sigma$-finite measures equivalent to $P$.

3.1. Construction. Take sequences $\left(p_{t}\right)_{t \geq 1}$ and $\left(\varepsilon_{t}\right)_{t \geq 1}$ such that

$$
\begin{gathered}
p_{1}>p_{2}>\cdots>0, \quad p_{t} \rightarrow 0(\text { as } t \rightarrow \infty), \quad \sum_{t=1}^{\infty} p_{t}=\infty, \\
\varepsilon_{1}>\varepsilon_{2}>\cdots>0, \quad \sum_{t=1}^{\infty} \varepsilon_{t}<\infty,
\end{gathered}
$$


and write

$$
\eta_{t}=\sum_{u=t}^{\infty} \varepsilon_{u}
$$

Let $\lambda_{1}$ be an arbitrary positive number $>1, n_{1}$ be an arbitrary integer $>1$ and $m_{1}$ be an arbitrary integer $>1+n_{1}$. Let

$$
M_{0}=1, \quad N_{1}=M_{0}+n_{1}, \quad M_{1}=N_{1}+m_{1} .
$$

We assume that $\left(\lambda_{u}, n_{u}, m_{u}\right), u=1,2, \ldots, t-1$ with $\lambda_{u-1}>\lambda_{u}>1, m_{u}>N_{u}$ $(1 \leq u \leq t-1)$ are chosen.

First step: Choice of $\lambda_{t}$. Take $\lambda_{t}$ such that

and

$$
1<\lambda_{t}<\lambda_{t-1}
$$

$$
\left(2 \lambda_{t} /\left(1+\lambda_{t}\right)\right)^{M_{t-1}}<\lambda_{t}^{M_{t-1}}<\exp \left(\varepsilon_{t}\right)
$$

Take $\rho_{t}>0$ such that

$$
1<\left(\lambda_{1}\right)^{2 M_{t-1}}<\left(\lambda_{t}\right)^{o_{t}} .
$$

Second step: Choice of $n_{t}$. Take $c_{t}>0$ such that

$$
\frac{1}{(2 \pi)^{\frac{T}{2}}} \int_{-c_{t}}^{c_{t}} \exp \left(-s^{2} / 2\right) d s=p_{t}
$$

It follows from the central limit theorem that one can obtain a large integer $n_{t}>M_{t-1}$ such that

where

$$
\begin{gathered}
\sum_{U_{t}-2 \rho_{t} \leq\left|k-n_{t} \lambda_{t} /\left(1+\lambda_{t}\right)\right| \leq U_{t}} f_{t}(k) \leq \frac{1}{4} p_{t}, \\
1-2 p_{t}<\sum_{\left|k-n_{t} \lambda_{t} /\left(1+\lambda_{t}\right)\right|>U_{t}} f_{t}(k) \leq 1-\frac{1}{2} p_{t},
\end{gathered}
$$

and

$$
f_{t}(k)=\left(1 /\left(1+\lambda_{t}\right)\right)^{n_{t}-k}\left(\lambda_{t} /\left(1+\lambda_{t}\right)\right)^{k}\left(\begin{array}{l}
n_{t} \\
k
\end{array}\right)
$$

$$
U_{t}=n_{t}^{\frac{1}{2}} \lambda_{t}^{\frac{1}{2}} c_{t} /\left(1+\lambda_{t}\right)
$$

for $k=0,1, \ldots, n_{t}$.

By (9) and (10) we have

$$
\sum_{\left|k-n_{t} \lambda_{t} /\left(1+\lambda_{t}\right)\right|<U_{t}-2 \rho_{t}} f_{t}(k)>\frac{1}{4} p_{t}
$$

Last step: Choice of $m_{t}$. We write

$$
F_{t}(\omega)=\prod_{u=1}^{t}\left(\frac{2}{1+\lambda_{u}}\right)^{n_{u}}\left(\lambda_{u}\right)^{\omega_{N_{t}-N_{u}+1}+\omega_{N_{t}-N_{u}+2}+\cdots+\omega_{N_{t}-M_{u-1}},}
$$

and for $R<S$

$$
H(\omega)=\chi[R, S]\left(\omega_{1}+\omega_{2}+\cdots+\omega_{n_{t}}\right)
$$

for $\omega \in \Omega$. It follows from Birkhoff's ergodic theorem that

$$
\lim _{m \rightarrow \infty} \frac{\sum_{j=0}^{m} F_{t}\left(T^{j} \omega\right) H\left(T^{j} \omega\right)}{\sum_{j=0}^{m} F_{t}\left(T^{j} \omega\right)}=\frac{E_{Q}\left(F_{t} H\right)}{E_{Q}\left(F_{t}\right)},
$$


$Q$-a.e. $\omega$, where $E_{Q}$ is the integration with respect to the measure $Q$.

Since

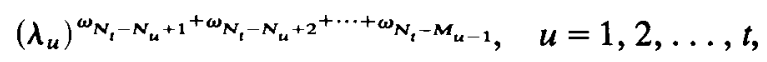

are independent random variables with respect to the measure $Q$, we have

$$
\begin{aligned}
E_{Q}\left(F_{t}\right) & =\prod_{u=1}^{t}\left(\frac{2}{1+\lambda_{u}}\right)^{n_{u}} \int\left(\lambda_{u}\right)^{\omega_{N_{t}-N_{u}+1}+\omega_{N_{t}-N_{u}+2}+\cdots+\omega_{N_{t}-M_{u-1}}} d Q(\omega) \\
& =\prod_{u=1}^{t} 1 \\
& =1
\end{aligned}
$$

and

$$
\begin{aligned}
E_{Q}\left(F_{t} H\right)= & \prod_{u=1}^{t-1}\left(\frac{2}{1+\lambda_{u}}\right)^{n_{u}} \int\left(\lambda_{u}\right)^{\omega_{N_{t}-N_{u}+1}+\omega_{N_{t}-N_{u}+2}+\cdots+\omega_{N_{t}-M_{u-1}} d Q(\omega)} \\
& \times\left(\frac{2}{1+\lambda_{t}}\right)^{n_{t}} \int_{R \leq \omega_{1}+\omega_{2}+\cdots+\omega_{n_{t}} \leq S}\left(\lambda_{t}\right)^{\omega_{1}+\omega_{2}+\cdots+\omega_{n_{t}}} d Q(\omega) \\
= & \sum_{R \leq k \leq S} f_{t}(k) .
\end{aligned}
$$

We write

$$
H_{t}(\omega)=\chi_{\left[n_{t} \lambda_{t} /\left(1+\lambda_{t}\right)-U_{t}, n_{t} \lambda_{t} /\left(1+\lambda_{t}\right)+U_{t}\right]}\left(\omega_{1}+\omega_{2}+\cdots+\omega_{n_{t}}\right),
$$

then we have from (10) and (16)

$$
E_{Q}\left(F_{t} H_{t}\right)<2 p_{t}
$$

It follows from (14), (15) and (18) that we have for all large integers $m$

$$
Q\left(\omega: \frac{\sum_{j=0}^{m-1} F_{t}\left(T^{j} \omega\right) H_{t}\left(T^{j} \omega\right)}{\sum_{j=0}^{m-1} F_{t}\left(T^{j} \omega\right)}<2 p_{t}\right)>1-\varepsilon_{t} .
$$

We take a large integer $m_{t}$ with

$$
m_{t}>N_{t}
$$

such that for

$$
A_{t}=\left\{\omega \in \Omega: \frac{\sum_{j=0}^{m_{t}-N_{t}-1} F_{t}\left(T^{j} \omega\right) H_{t}\left(T^{j} \omega\right)}{\sum_{j=0}^{m_{t}-N_{t}-1} F_{t}\left(T^{j} \omega\right)}<2 p_{t}\right\}
$$

we have

$$
Q\left(A_{t}\right)>1-\varepsilon_{t}
$$

and

$$
\frac{N_{t} \exp \left(2 \eta_{t+1}\right)\left(\lambda_{1}\right)^{3 N_{t}}}{m_{t}-N_{t}}<\frac{1}{2} \varepsilon_{t}
$$

THEOREM 2. Let $T$ be the shift on the infinite product measure space

$$
(\Omega, P)=\left(\prod_{k=-\infty}^{\infty}\{0,1\}, \prod_{k=-\infty}^{\infty} P_{k}\right)
$$

constructed above. Then $T$ is non-singular, ergodic and admits no $\sigma$-finite invariant measure equivalent to $P$. 
After some preparation we shall prove this theorem.

3.2. Radon-Nikodym density $(d P T / d P)(\omega)$. The sequence $\left(\lambda_{t}\right)_{t \geq 1}$ in $\S 3.1$ satisfies the non-singularity condition ( 3 )

$$
\begin{aligned}
\sum_{t=1}^{\infty}\left(\log \lambda_{t}\right)^{2} & <\sum_{t=1}^{\infty} \varepsilon_{t}^{2} \quad(\text { by }(7)) \\
& <\infty \quad(\text { by }(5)) .
\end{aligned}
$$

Thus the shift $T$ constructed in $\S 3.1$ is non-singular.

LEMMA 1. Let $T$ be the shift in $\$ 3.1$ and put

$$
K_{t, i}(\omega)=\prod_{u=t+1}^{\infty}\left(\lambda_{u}\right)^{-\left\{\omega_{-N_{u}+1}+\omega_{-N_{u}+2}+\cdots+\omega_{-N_{u}+i}\right\}+\left\{\omega_{-M_{u-1}+1}+\omega_{-M_{u-1}+2}+\cdots+\omega_{-M_{u-1}+i}\right\}},
$$

then we have

and

$$
\frac{d P T^{i}}{d P}(\omega)=K_{t, i}(\omega) \times \prod_{k=-N_{t}+1}^{i-1} \frac{P_{k-i}\left(\omega_{k}\right)}{P_{k}\left(\omega_{k}\right)} \quad \text { if } 0 \leq i<N_{t},
$$

$$
\begin{aligned}
\frac{d P T^{i}}{d P}(\omega)= & K_{t, i}(\omega) \times \prod_{u=1}^{t}\left(\frac{1+\lambda_{u}}{2}\right)^{n_{u}}\left(\lambda_{u}\right)^{-\left\{\omega_{-N_{u}+1}+\omega_{-N_{u}+2}+\cdots+\omega_{-M_{u-1}}\right\}} \\
& \times F_{t}\left(T^{i-N_{i}} \omega\right) \text { if } N_{t} \leq i<m_{t}
\end{aligned}
$$

for $P$-a.e. $\omega$, where $F_{t}(\omega)$ is the random variable defined in (12).

Proof. For $0<i<N_{t}$ it follows from (2) that

$$
\begin{aligned}
& \frac{d P T^{i}}{d P}(\omega)=\prod_{u=t+1}^{\infty}\left\{\prod_{k=-N_{u}+1}^{-N_{u}+i}\left(\frac{P_{k-i}\left(\omega_{k}\right)}{P_{k}\left(\omega_{k}\right)}\right) \times \prod_{k=-M_{u-1}+1}^{-M_{u-1}+i}\left(\frac{P_{k-i}\left(\omega_{k}\right)}{P_{k}\left(\omega_{k}\right)}\right)\right\} \\
& \times \prod_{k=-N_{t}+1}^{i-1} \frac{P_{k-i}\left(\omega_{k}\right)}{P_{k}\left(\omega_{k}\right)} \\
& =\prod_{u=t+1}^{\infty}\left\{\left(\frac{1+\lambda_{u}}{2}\right)^{i} \times\left(\lambda_{u}\right)^{-\left\{\omega_{-N_{u}+1}+\omega_{-N_{u}+2}+\cdots+\omega_{-N_{u}+i}\right\}}\right.
\end{aligned}
$$

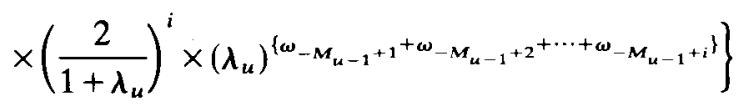

$$
\begin{aligned}
& \times \prod_{k=-N_{i}+1}^{i-1} \frac{P_{k-i}\left(\omega_{k}\right)}{P_{k}\left(\omega_{k}\right)}
\end{aligned}
$$

for $P$-a.e. $\omega$.

For $N_{t} \leq i<m_{t}$, the second factor of (23) is

$$
\begin{aligned}
& \prod_{u=1}^{t}\left\{\prod_{k=-N_{u}+1}^{-M_{u-1}} \frac{P_{k-i}\left(\omega_{k}\right)}{P_{k}\left(\omega_{k}\right)}\right\} \times \prod_{u=1}^{t}\left\{\prod_{k=i-N_{u}+1}^{i-M_{u-1}} \frac{P_{k-i}\left(\omega_{k}\right)}{P_{k}\left(\omega_{k}\right)}\right\} \\
&=\prod_{u=1}^{t}\left\{\left(\frac{1+\lambda_{u}}{2}\right)^{n_{u}}\left(\lambda_{u}\right)^{-\left\{\omega_{-N_{u}+1}+\omega_{-N_{u}+2}+\cdots+\omega_{-M_{u-1}}\right\}}\right\} \\
& \times \prod_{u=1}^{t}\left\{\left(\frac{2}{1+\lambda_{u}}\right)^{n_{u}}\left(\lambda_{u}\right)^{\left\{\omega_{i-N_{u}+1}+\omega_{i-N_{u}+2}+\cdots+\omega_{i-M_{u-1}}\right\}}\right\}
\end{aligned}
$$

for $P$-a.e. $\omega$. 
3.3. Ratio ergodic theorem. Krengel proved in [3] that if the shift on an infinite product measure

$$
\left(\prod_{k=-\infty}^{\infty}\{0,1\}, \prod_{k=-\infty}^{\infty} P_{k}\right)
$$

with

$$
P_{k}(0)=P_{k}(1)=\frac{1}{2} \quad \text { for } k>0
$$

is non-singular and conservative then it is ergodic. The shift in $\S 3.1$ satisfies the condition. This is because

$$
F_{t}(\omega)>\prod_{u=1}^{i}\left(\frac{2}{1+\lambda_{u}}\right)^{n_{u}}
$$

for every $\omega$, so for $N_{t} \leq i<m_{t}$ we have from (24)

$$
\begin{aligned}
\frac{d P T^{i}}{d P}(\omega) & \geq \prod_{u=t+1}^{\infty}\left(\lambda_{u}\right)^{-(i+1)} \times \prod_{u=1}^{t}\left(\lambda_{u}\right)^{-n_{u}} \\
& \geq \prod_{u=t+1}^{\infty} \lambda_{u}^{-M_{u-1}} \times \prod_{u=1}^{t} \lambda_{1}^{-n_{u}} \\
& \geq \prod_{u=t+1}^{\infty} \exp \left(-\varepsilon_{u}\right) \times\left(\lambda_{1}\right)^{-N_{t}} \quad(\text { by }(7)) \\
& =\exp \left(-\eta_{t+1}\right) \times\left(\lambda_{1}\right)^{-N_{t}}
\end{aligned}
$$

Then we have for a.e. $\omega$,

$$
\begin{aligned}
\sum_{i=0}^{\infty} \frac{d P T^{i}}{d P}(\omega) & \geq \sum_{t=1}^{\infty} \sum_{i=N_{t}}^{m_{t}-1} \frac{d P T^{i}}{d P}(\omega) \\
& \geq \sum_{t=1}^{\infty}\left(m_{t}-N_{t}\right) \exp \left(-\eta_{t+1}\right)\left(\lambda_{1}\right)^{-N_{t}} \\
& \geq \sum_{t=1}^{\infty} \frac{2 N_{t} \exp \left(\eta_{t+1}\right)\left(\lambda_{1}\right)^{2 N_{t}}}{\varepsilon_{t}} \quad(\text { by }(22)) \\
& =\infty .
\end{aligned}
$$

It follows from the Chacon-Ornstein ratio ergodic theorem [1] that for any measurable set $E$ with $P(E)>0$,

$$
\lim _{n \rightarrow \infty} \frac{\sum_{i=0}^{n-1} \frac{d P T^{i}}{d P}(\omega) \chi_{E}\left(T^{i} \omega\right)}{\sum_{i=0}^{n-1} \frac{d P T^{i}}{d P}(\omega)}=P(E)
$$

for $P$-a.e. $\omega$.

Proposition 1. Let $T$ be the shift constructed in $\S 3.1$ and, for a measurable set $E$ with $P(E)>0$ and $t>1$, we write

$$
\mathrm{I}_{t}(\omega)=\frac{\sum_{i=0}^{m_{\mathrm{t}}-1} \frac{d P T^{i}}{d P}(\omega)_{\chi E}\left(T^{i} \omega\right)}{\sum_{i=0}^{m_{\mathrm{t}}-1} \frac{d P T^{i}}{d P}(\omega)},
$$




$$
\begin{aligned}
& \mathrm{II}_{t}(\omega)=\frac{\sum_{i=N_{t}}^{m_{t}-1} \frac{d P T^{i}}{d P}(\omega) \chi_{E}\left(T^{i} \omega\right)}{\sum_{i=N_{t}}^{m_{t}-1} \frac{d P T^{i}}{d P}(\omega)}, \\
& \operatorname{III}_{t}(\omega)=\frac{\sum_{i=N_{t}}^{m_{t}-1} F_{t}\left(T^{i-N_{t}} \omega\right)_{\chi_{E}}\left(T^{i} \omega\right)}{\sum_{i=N_{t}}^{m_{t}-1} F_{t}\left(T^{i-N_{t}} \omega\right)}, \\
& \operatorname{IV}_{t}(\omega)=\frac{\sum_{i=N_{t}}^{m_{t}-1} F_{t}\left(T^{i-N^{t}} \omega\right)\left\{1-H_{t}\left(T^{i-N_{\mathrm{t}}} \omega\right)\right\}_{\chi_{E}}\left(T^{i} \omega\right)}{\sum_{i=N_{t}}^{m_{\mathrm{t}}-i} F_{t}\left(T^{i-N_{\mathrm{t}}} \omega\right)},
\end{aligned}
$$

where $H_{t}(\omega)$ is the random variable defined in (17). Then we have

$$
\lim _{t \rightarrow \infty} \mathrm{I}_{t}(\omega)=\lim _{t \rightarrow \infty} \mathrm{II}_{t}(\omega)=\lim _{t \rightarrow \infty} \mathrm{III}_{t}(\omega)=\lim _{t \rightarrow \infty} \operatorname{IV}_{t}(\omega)=P(E)
$$

for $P$-a.e. $\omega$.

Proof. We already mentioned in (26) that the limit of $\left(I_{t}\right)_{t \geq 1}$ exists for $P$-a.e. $\omega$, and is equal to the constant $P(E)$. Since

$$
\begin{aligned}
\left|\mathrm{I}_{t}(\omega)-\mathrm{II}_{t}(\omega)\right| & \leq 2 \times \frac{\sum_{i=0}^{N_{t}-1} \frac{d P T^{i}}{d P}(\omega)}{\sum_{i=N_{t}-1} \frac{d P T^{i}}{d P}(\omega)} \\
& \leq 2 \times \frac{N_{t} \exp \left(\eta_{t+1}\right) \lambda_{1}^{2 N_{t}}}{\left(m_{t}-N_{t}\right) \exp \left(-\eta_{t+1}\right) \lambda_{1}^{-N_{t}}} \quad(\text { by }(23) \text { and }(25)) \\
& <\varepsilon_{t} \quad(\text { by }(22)),
\end{aligned}
$$

we have

$$
\lim _{m \rightarrow \infty} I_{t}(\omega)=\lim _{m \rightarrow \infty} I_{t}(\omega)
$$

for $P$-a.e. $\omega$.

It follows from (24) that

$$
\mathrm{II}_{t}(\omega)=\frac{\sum_{i=N_{t}}^{m_{t}-1} K_{t, i}(\omega) F_{t}\left(T^{i-N_{t}} \omega\right) \chi_{E}\left(T^{i} \omega\right)}{\sum_{i=N_{t}}^{m_{t}-1} K_{t, i}(\omega) F_{t}\left(T^{i-N_{i}} \omega\right)}
$$

Since

$$
\exp \left(-\eta_{t+1}\right)<K_{t, i}(\omega)<\exp \left(\eta_{t+1}\right),
$$

we have

$$
\exp \left(-2 \eta_{t+1}\right)<\frac{\mathrm{II}_{t}(\omega)}{\mathrm{III}_{t}(\omega)}<\exp \left(2 \eta_{t+1}\right)
$$

Thus

$$
\lim _{t \rightarrow \infty} \mathrm{II}_{t}(\omega)=\lim _{t \rightarrow \infty} \operatorname{III}_{t}(\omega)
$$

for $P$-a.e. $\omega$. 
Let us reconsider the set $A_{t}$ defined in (21). Since this set is $\bigvee_{k=1}^{M_{t}-1} \mathscr{F}_{k}$-measurable, we have

$$
P\left(A_{t}\right)=Q\left(A_{t}\right)>1-\varepsilon_{t}
$$

Since

$$
\sum_{t=1}^{\infty} P\left(A_{t}^{c}\right) \leq \sum_{t=1}^{\infty} \varepsilon_{t}<\infty
$$

by the Borel-Cantelli lemma (in the general case), it holds that $P$-a.e. $\omega$ is in $A_{t}$ for all but a finite number of $t$. This means that for $P$-a.e. $\omega$ and for all large numbers $t$

$$
\begin{aligned}
\frac{\sum_{i=N_{t}}^{m_{t}-1} F_{t}\left(T^{i-N_{t}} \omega\right) H_{t}\left(T^{i-N_{t}} \omega\right) \chi_{E}\left(T^{i} \omega\right)}{\sum_{i=N_{t}}^{m_{t}-1} F_{t}\left(T^{i-N_{t}} \omega\right)} & \leq \frac{\sum_{j=0}^{m_{t}-N_{t}-1} F_{t}\left(T^{j} \omega\right) H_{t}\left(T^{j} \omega\right)}{\sum_{j=0}^{m_{t}-N_{t}-1} F_{t}\left(T^{j} \omega\right)} \\
& <2 p_{t} .
\end{aligned}
$$

Since $p_{t}$ converges to 0 , we have that for $P$-a.e. $\omega$

$$
\begin{aligned}
\lim _{i \rightarrow \infty} \operatorname{III}_{t}(\omega) & =\lim _{t \rightarrow \infty} \frac{\sum_{i=N_{t}}^{m_{t}-1} F_{t}\left(T^{i-N_{t}} \omega\right) H_{t}\left(T^{i-N_{t}} \omega\right) \chi_{E}\left(T^{i} \omega\right)}{\sum_{i=N_{t}}^{m_{t}-1} F_{t}\left(T^{i-N_{t}} \omega\right)}+\lim _{t \rightarrow \infty} \operatorname{IV}_{t}(\omega) \\
& =\lim _{t \rightarrow \infty} \operatorname{IV}_{t}(\omega) .
\end{aligned}
$$

\subsection{Recurrence}

Proposition 2. Let $T$ be the shift constructed in $\S 3.1$. For any measurable set $E$ with $P(E)>0$, there exist for $P$-a.e. $\omega$ an infinite number of $t$ such that $T^{i} \omega \in E$ for some $i, N_{t} \leq i<m_{t}$, and that for such $i$, either

$$
\frac{d P T^{i}}{d P}(\omega)>\left(\lambda_{1}\right)^{2 M_{t-1}} \exp \left(-\eta_{t+1}\right)
$$

or

$$
\frac{d P T^{i}}{d P}(\omega)<\left(\lambda_{1}\right)^{-2 M_{t-1}} \exp \left(\eta_{t+1}\right)
$$

holds.

Proof. We define a set $B_{t}$ by

$$
B_{t}=\left\{\omega \in \Omega: \frac{n_{t} \lambda_{t}}{1+\lambda_{t}}-U_{t}+2 \rho_{t}<\omega_{-N_{t}+1}+\omega_{-N_{t}+2}+\cdots+\omega_{-M_{t-1}}<\frac{n_{t} \lambda_{t}}{1+\lambda_{t}}+U_{t}-2 \rho_{t}\right\} .
$$

By (11) we have

$$
P\left(B_{t}\right)>\frac{1}{4} p_{t}
$$

Since the set $B_{t}$ is $V_{j=-N_{t}+1}^{-M_{r-1}} \mathscr{F}_{j}$-measurable, the sets $B_{1}, B_{2}, \ldots$ are independent with respect to the measure $P$. It follows from the Borel-Cantelli lemma (in the independent case) that for $P$-a.e. $\omega$ there exists an infinite number of $t$ such that $\omega \in B_{t}$. 
On the other hand we have from proposition 1 that

$$
\lim _{t \rightarrow \infty} \frac{\sum_{i=N_{t}}^{m_{t}-1} F_{t}\left(T^{i-N_{t}} \omega\right)\left\{1-H_{t}\left(T^{i-N_{t}} \omega\right)\right\}_{\chi_{E}}\left(T^{i} \omega\right)}{\sum_{i=N_{t}}^{m_{t}-1} F_{t}\left(T^{i-N_{t}} \omega\right)}=P(E)
$$

for $P$-a.e. $\omega$. Then we have that for $P$-a.e. $\omega$ and for all large numbers $t, T^{i} \omega \in E$, for some $i$ with $N_{t} \leq i<m_{t}$, and that for such $i$ either

$$
\omega_{i-N_{t}+1}+\omega_{i-N_{t}+2}+\cdots+\omega_{i-M_{t-1}}>\frac{n_{t} \lambda_{t}}{1+\lambda_{t}}+U_{t}
$$

or

$$
\omega_{i-N_{t}+1}+\omega_{i-N_{t}+2}+\cdots+\omega_{i-M_{t-1}}<\frac{n_{t} \lambda_{t}}{1+\lambda_{t}}-U_{t}
$$

holds.

Combining these results, we see that there exists for $P$-a.e. $\omega$ an infinite number of $t$ such that $T^{i} \omega \in E$ for some $i$ with $N_{t} \leq i<m_{t}$, and that for such $i$,

$$
\left(\lambda_{t}\right)^{\left\{\omega_{i-N_{t}+1}+\omega_{i-N_{t}+2}+\cdots+\omega_{i-M_{t-1}}\right\}-\left\{\omega_{-N_{t}+1}+\omega_{-N_{t}+2}+\cdots+\omega_{-M_{t-1}}\right\}}>\left(\lambda_{t}\right)^{2 \rho_{t}}
$$

or

$$
\left(\lambda_{t}\right)^{\left\{\omega_{i-N_{t}+1}+\omega_{i-N_{t}+2}+\cdots+\omega_{i-M_{t-1}}\right\}-\left\{\omega_{-N_{t}+1}+\omega_{-N_{t}+2}+\cdots+\omega_{-M_{t-1}}\right\}}<\left(\lambda_{t}\right)^{-2 \rho_{t}}
$$

holds. If (28) holds then it follows from (24) that

$$
\begin{aligned}
\frac{d P T^{i}}{d P}(\omega)> & \left(\lambda_{t}\right)^{\left\{\omega_{i-N_{t}+1}+\omega_{i-N_{t}+2}+\cdots+\omega_{i-M_{t-1}}\right\}-\left\{\omega_{-N_{t}+1}+\omega_{-N_{t}+2}+\cdots+\omega_{-M_{t-1}}\right\}} \\
& \times\left(\lambda_{1}\right)^{-2 M_{t-1}} \exp \left(-\eta_{t+1}\right) \\
> & \left(\lambda_{t}\right)^{2 \rho_{t}}\left(\lambda_{1}\right)^{-2 M_{t-1}} \exp \left(-\eta_{t+1}\right) \\
> & \left(\lambda_{1}\right)^{2 M_{t-1}} \exp \left(-\eta_{t+1}\right) \quad(\text { by }(8)) .
\end{aligned}
$$

If (29) holds then it follows from (24) that

$$
\begin{aligned}
\frac{d P T^{i}}{d P}(\omega)< & \left(\lambda_{t}\right)^{\left\{\omega_{i-N_{t}+1}+\omega_{i-N_{t}+2}+\cdots+\omega_{i-M_{t-1}}\right\}-\left\{\omega_{-N_{t}+1}+\omega_{-N_{t}+2}+\cdots+\omega_{-M_{t-1}}\right\}} \\
& \times\left(\lambda_{1}\right)^{2 M_{t-1}} \exp \left(\eta_{t+1}\right) \\
< & \left(\lambda_{t}\right)^{-2 \rho_{t}}\left(\lambda_{1}\right)^{2 M_{t-1}} \exp \left(\eta_{t+1}\right) \\
< & \left(\lambda_{1}\right)^{-2 M_{t-1}} \exp \left(-\eta_{t+1}\right)
\end{aligned}
$$

3.5. Proof of theorem 2. We assume that $T$ admits a $\sigma$-finite invariant measure equivalent to $P$. Then there exists a positive measurable function $f(\omega)$ such that

$$
\frac{d P T^{i}}{d P}(\omega)=\frac{f\left(T^{i} \omega\right)}{f(\omega)}
$$

for $i \in \mathbb{Z}$ and $P$-a.e. $\omega$. Take $a>b>0$ such that

$$
P(\omega \in \Omega: b<f(\omega)<a)>0
$$

and set $E=\{\omega \in \Omega: b<f(\omega)<a\}$. Then we have

$$
\frac{b}{a}<\frac{d P T_{E}^{i}}{d P}<\frac{a}{b}
$$


for all $i \in \mathbb{Z}$ and $P$-a.e. $\omega$, that is, the functions $\left(d P T_{E}^{i} / d P\right)(\omega), i \in \mathbb{Z}$, have a uniformly positive lower bound and a uniform upper bound, where $T_{E}$ is the induced transformation on the set $E$ of $T$. However, this contradicts proposition 2.

Acknowledgement. The author would like to thank W. Krieger for calling his attention to U. Krengel's paper [3], and M. Osikawa and Y. Ito for valuable discussions. He also would like to thank the Institut für Angewandte Mathematik, Universität Heidelberg, and Le Centre de Physique Théorique, CNRS, Marseille, for their hospitality while part of this work was done. The research was supported in part by the Alexander von Humboldt Stiftung.

\section{REFERENCES}

[1] R. V. Chacon \& D. S. Ornstein. A general ergodic theorem. Illinois J. Math. 4 (1960), 153-160.

[2] S. Kakutani. On equivalence of infinite product measures. Ann. Math. 49 (1948), 214-224.

[3] U. Krengel. Transformations without finite invariant measure have finite strong generators. Lecture Notes in Math. no. 160, pp. 135-157. Springer-Verlag: Berlin, 1970. 